\title{
NCCN Guidelines Updates: Breast Cancer
}

\author{
Presented by Sharon H. Giordano, MD, MPH; Anthony D. Elias, MD; and William J. Gradishar, MD
}

\begin{abstract}
The emergence of CDK4/6 inhibitors has changed the treatment algorithm for advanced/metastatic estrogen receptor-positive breast cancer. In pivotal trials of palbociclib, ribociclib, and abemaciclib, doubling in progression-free survival has been seen. All 3 agents in this class are now included in the NCCN Guidelines for Breast Cancer, and clinicians should be incorporating these agents into their treatment algorithms. The other important issue in this breast cancer setting is extended duration of endocrine therapy. Most of the benefit is modest and toxicity is an issue; therefore, extended-duration endocrine therapy should be highly individualized. For triple-negative disease, platinum agents and PARP inhibitors are helping some patients, but immunotherapies and other novel classes of drugs now in development hold the promise of even better outcomes. In HER2-positive early-stage disease, dual HER2 blockade is of modest benefit, and extended treatment with neratinib may be a good option for some high-risk patients.
\end{abstract}

J Natl Compr Canc Netw 2018;16(5.5):605-610 doi: 10.6004/jncen.2018.0043

\section{Panel Refines Important Breast Cancer Recommendations}

In the absence of substantive changes to the NCCN Guidelines for Breast Cancer this year, members of the NCCN panel discussed refinements to the recommendations, described the use of recently approved treatments, and highlighted agents in development that should answer some unmet needs.

Sharon H. Giordano, MD, MPH, Professor of Breast Medical Oncology and the Hubert L. and Olive Stringer Distinguished Professor in Cancer Research,

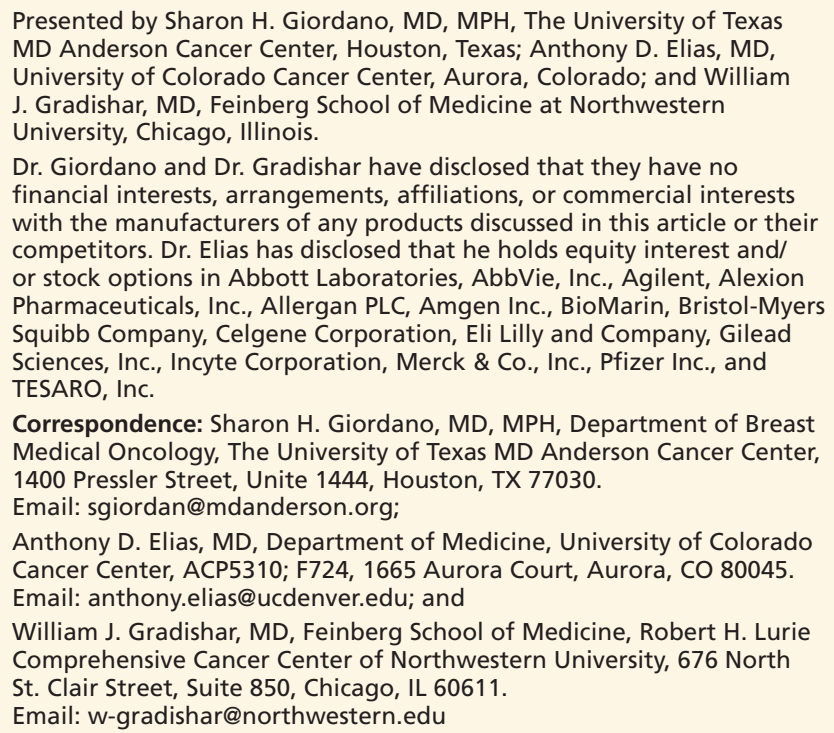

The University of Texas MD Anderson Cancer Center, described treatments for patients with HER2-positive cancer. Anthony D. Elias, MD, Professor of Medicine, and the Martha Cannon Dear Chair in Breast Cancer Research, University of Colorado Cancer Center, presented new developments in triple-negative breast cancer (TNBC). William J. Gradishar, MD, the Betsy Bramsen Professor of Breast Oncology and Professor of Medicine, Feinberg School of Medicine, Northwestern University, reviewed the latest recommendations for advanced hormone-sensitive breast cancer at the NCCN 23rd Annual Conference.

\section{New Adjuvant Therapy Options for Patients With HER2-Positive Cancer}

Two new adjuvant treatment options for patients with HER2-positive disease are in the 2018 NCCN Guidelines. The APHINITY trial of 4,805 patients added to the body of data for dual HER2 inhibition by showing a positive_-albeit small—benefit for adding pertuzumab to trastuzumab and chemotherapy. The absolute benefit in preventing invasive disease was $0.9 \%$ at 3 years and $1.7 \%$ at 4 years (hazard ratio [HR], $0.81 ; P=.045$ ), with slightly more benefit shown in the hormone receptornegative and node-positive subsets. ${ }^{1}$ Based on the results of this trial, the FDA granted approval for adjuvant pertuzumab. 
Also for patients with HER2-positive disease, neratinib was approved based on results of the ExteNET trial that included 2,840 women. ${ }^{2}$ Patients who received neratinib after completing 1 year of trastuzumab had an absolute benefit of $2.3 \%$ in invasive disease-free survival (DFS). The drug seemed to be of more value in the hormone receptor-positive versus - negative subset.

The main adverse event with neratinib is diarrhea, which was seen in $95 \%$ of patients, including $40 \%$ with grade 3 toxicity. When patients follow a protocol for preventing and managing diarrhea, this side effect can be greatly diminished, Dr. Giordano indicated.

"You can consider extended anti-HER2 treatment with neratinib in HER2-positive, hormone receptor-positive patients you deem at high risk for recurrence, although it's kind of difficult for this drug to find its place since most patients at high risk will have received dual targeted therapy with trastuzumab and pertuzumab," she said.

\section{Small HER2-Positive Tumors}

No single standard treatment exists for patients with small, node-negative, HER2-positive breast cancers, but there is evidence that chemotherapy might be useful in some. Tolaney et $\mathrm{al}^{3}$ conducted a study of adjuvant paclitaxel (given weekly for 12 weeks with trastuzumab) plus trastuzumab monotherapy (for 9 months) in 406 patients with tumors $\leq 3 \mathrm{~cm}$. The recurrence-free survival rate for these stage T1, nodenegative tumors was $99.2 \%$ at 3 years, $98.1 \%$ at 5 years, and $97.5 \%$ at 7 years in an update presented at the 2017 ASCO Annual Meeting. ${ }^{4}$

\section{New Developments in TNBC}

Dr. Elias updated attendees on new developments in TNBC, for which there is only one approved targeted agent - the oral poly(adenosine diphosphateribose) polymerase (PARP) inhibitor olaparib. With only chemotherapy as an approved treatment option, "There is clearly an unmet need for targeted therapies in triple-negative disease," he said.

For metastatic disease, Dr. Elias made the following key points:

- First-line chemotherapy should be with a taxane or anthracycline if not previously used (in the neoadjuvant or adjuvant setting). Weekly paclitaxel is generally the preferred taxane.

- Sequential single agents are preferred unless there is visceral crisis. Combinations yield higher response rates but do not improve overall survival.

- Eribulin and capecitabine and platins are likely more effective than gemcitabine and vinorelbine, but line of therapy may be more predictive of response.

- Chemotherapy resistance develops quickly, and third and fourth lines of therapy offer little benefit.

\section{Platins, PARP Inhibitors}

Platinum agents and PARP inhibitors are effective drugs in patients with BRCA mutations, which occur in approximately $30 \%$ of TNBC cases. In the neoadjuvant setting, single-agent cisplatin resulted in a $61 \%$ pathologic complete response rate in patients with BRCA mutations, including those with TNBC. ${ }^{5}$ In the metastatic setting, the TNT trial provided no evidence of greater benefit with carboplatin compared with docetaxel in unselected patients with advanced TNBC, but the BRCA-mutated subset had a doubling in response and longer progression-free survival (PFS). ${ }^{6}$ In a phase III trial of patients with metastatic disease, olaparib monotherapy doubled the response rate and improved median PFS from 4.2 to 7.0 months compared with standard therapy (HR, 0.58; $P<.001) .{ }^{7}$ Similar data now exist for talazoparib.

Olaparib is approved for previously treated metastatic breast cancer with germline BRCA mutations, but not for patients with somatic mutations or low expression of BRCA. "We don't yet know about efficacy in somatic mutations, but we do know the germline tumors benefit," he said.

"The question now is whether we should test everyone with metastatic breast cancer, since the assays are relatively cheap and olaparib is an active therapeutic agent. No survival advantage, however, has been documented," Dr. Elias said.

\section{Immune Checkpoint Inhibitors in TNBC}

TNBC appears to be a fairly good target for immunotherapies, an approach that has been studied in both heavily pretreated and treatment-naïve patients. Response rates have ranged from $5 \%$ to $19 \%$ in refractory patients, but are higher in treatment-naïve pa- 
tients. In studies of pembrolizumab and atezolizumab as first-line agents, responses were seen in $23 \%$ and $26 \%$, respectively. ${ }^{8,9}$ In the atezolizumab study, duration of response was 8 to 26 months. ${ }^{9}$

As neoadjuvant therapy, the addition of pembrolizumab "markedly improved" pathologic complete response rates in the TNBC and estrogen receptor (ER)-positive cohorts of the I-SPY trial. ${ }^{10}$ In patients with TNBC, the probability of pathologic complete response increased to $60 \%$ versus $20 \%$ with chemotherapy alone.

A number of immunotherapeutic strategies are being pursued in TNBC, including use as single agents (versus chemotherapy) and in combination with various treatments, some new and some traditional, including chemotherapy, radiotherapy, adenosine antagonists, and inhibitors of PARP, IDO, MEK, HDAC, and CTLA4. Immunologic agents are being evaluated in the adjuvant and neoadjuvant settings, including for their benefit in eradicating residual disease postoperatively.

\section{Promising Novel Approaches}

Many novel strategies are also being pursued for TNBC, including antibody-drug conjugates and androgen receptor antagonists. Antibody-drug conjugates in development target LIV-1 (a transmembrane zinc transporter expressed in almost $90 \%$ of TNBC), GPNMB (a gene expressed in aggressive breast cancers), Trop-2 (a cell-surface glycoprotein expressed on $>90 \%$ of TNBC), and more.

A phase I trial of 35 patients with TNBC showed a response rate of $37 \%$ with SGN-LIV1A. ${ }^{11}$ Glembatumumab vedotin has also shown significant activity in metastatic breast cancer, including in a phase II study of 42 patients in which median PFS was 17.9 weeks for TNBC and 18.0 weeks for GPNMB-positive tumors. ${ }^{12}$ In a subsequent study involving 124 heavily pretreated patients, an unplanned analysis by subgroup showed the response rate to be $18 \%$ with glembatumumab versus $0 \%$ with physician's choice in patients with TNBC and $40 \%$ and $0 \%$, respectively, in those with GPNMB-positive cancer. ${ }^{13}$

One antibody-drug conjugate that has already received breakthrough designation by the FDA is sacituzumab govitecan, which targets Trop-2. Sacituzumab govitecan has led to responses in at least $30 \%$ of heavily pretreated patients. ${ }^{14}$ Finally, agents targeting the androgen receptor, which is expressed by approximately half of patients with TNBC, look promising. In a phase II study of enzalutamide in 118 patients with androgen receptor-expressing disease who had been pretreated, responses were seen in $6 \%$, with $42 \%$ experiencing clinical benefit at 24 weeks. ${ }^{15}$

\section{Hormone-Sensitive Advanced Breast Cancer}

The NCCN Guidelines reflect some new standards for ER-positive breast cancer, primarily incorporating 3 inhibitors of cyclin-dependent kinases 4 and 6 (CDK4/6). In addition, there is continued discussion about the optimal duration of endocrine therapy. These topics were discussed by Dr. Gradishar.

Summing up the key revisions, Dr. Gradishar said, "We now have 3 CDK4/6 inhibitors in the guidelines-last year, there were only 2 -and we now have premenopausal data for their use. We've also added fulvestrant as endocrine therapy, reflecting the results of the FALCON trial. We have further addressed the extended duration of adjuvant endocrine therapy, with every permutation of it. Mostly, we state that endocrine therapy beyond 5 years can be considered, but it's not an absolute."

\section{CDK4/6 Inhibitors Are Game-Changers}

Optimizing the treatment of ER-positive breast cancer depends on overcoming resistance to endocrine agents. This tall effort has met with some success, with the availability of CDK4/6 inhibitors. "If you reflect what's gone on over the past couple of years, it's really been the era of the CDK4/6 inhibitors," Dr. Gradishar noted.

Three CDK4/6 inhibitors are approved in the metastatic setting for hormone receptor-positive/ HER2-negative disease, with these indications:

- Palbociclib: in combination with an aromatase inhibitor (AI) as initial therapy in postmenopausal women, and in combination with fulvestrant in patients with metastatic disease who progress on endocrine therapy.

- Ribociclib: in combination with an $\mathrm{AI}$ as initial therapy in postmenopausal women.

- Abemaciclib: for women and men as monotherapy in the setting of disease progression after endocrine therapy and prior chemotherapy, and as initial therapy in combination with an AI as initial endocrine therapy for postmenopausal women. 
"So you see it reflected in the guidelines that you have a number of different options among the 3 CDK4/6 inhibitors, with category 1 level evidence. Choosing which one, or in what setting to use them, is really based on the results of the clinical trials," he said.

The pivotal trial groups-PALOMA for palbociclib, MONALEESA for ribociclib, and MONARCH for abemaciclib-have "fairly striking consistency... favoring combinations over monotherapy with an AI alone," Dr. Gradishar noted. "To a large extent the results [Kaplan-Meier curves] are fairly superimposable....in most cases showing a doubling in PFS."

Unfortunately, it has not been possible to identify subsets who will not benefit from the addition of CDK4/6 inhibitors, as reported from an analysis of MONARCH 3 (Figure 1). ${ }^{16}$ "Benefit was seen in all subsets, but what was interesting was that the worse the prognosis, based on clinical features, the bigger the gain from the addition of the CDK4/6 inhibitor," he noted. "This may suggest there is a group who are particularly likely to benefit."

Data are available backing the use of this class in premenopausal patients as well, based on findings from the global MONALEESA-7 trial of almost 700 premenopausal or perimenopausal women rendered postmenopausal. ${ }^{17}$ Patients had received no prior endocrine therapy for metastatic disease; by physician's choice they received an AI (mostly) or tamoxifen, plus ribociclib or placebo. Similar to studies in postmenopausal patients, ribociclib led to a doubling in PFS, which was 13.0 months with placebo versus 23.8 months with ribociclib ( $\mathrm{HR}, 0.553$;

\begin{tabular}{|c|c|c|}
\hline & No & Yes \\
\hline Liver metastases & $\begin{array}{l}\text { HR, } 0.57(95 \% \mathrm{Cl}, 0.41-0.78) \\
\text { PFS, } 15.35 \mathrm{mo} \text { vs NR } \\
\mathrm{N}=135 \text { vs } 280\end{array}$ & $\begin{array}{l}\text { HR, } 0.47(95 \% \mathrm{Cl}, 0.25-0.87) \\
\text { PFS, } 7.2 \text { vs } 15.0 \mathrm{mo} \\
\mathrm{N}=30 \text { vs } 48\end{array}$ \\
\hline \multirow[t]{2}{*}{ Bone-only metastases } & $\begin{array}{l}\text { HR, } 0.51 \text { ( } 95 \% \text { Cl, } 0.38-0.70) \\
\text { PFS, } 11.7 \mathrm{mo} \text { vs NR } \\
\mathrm{N}=126 \text { vs } 258\end{array}$ & $\begin{array}{l}\text { HR, } 0.58(95 \% \mathrm{Cl}, 0.27-1.25) \\
\text { PFS, NR vs NR } \\
\mathrm{N}=39 \text { vs } 70\end{array}$ \\
\hline & Low/intermediate & High \\
\hline \multirow[t]{2}{*}{ Tumor grade } & $\begin{array}{l}\text { HR, } 0.63(95 \% \mathrm{Cl}, 0.44-0.92) \\
\mathrm{PFS}, 14.8 \mathrm{mo} \text { vs NR } \\
\mathrm{N}=96 \text { vs } 180\end{array}$ & $\begin{array}{l}\text { HR, } 0.39 \text { (95\% Cl, } 0.21-0.73) \\
\text { PFS, } 8.9 \text { mo vs NR } \\
N=32 \text { vs } 64\end{array}$ \\
\hline & $236 \mathrm{mo}$ & $<36 \mathrm{mo}$ \\
\hline Treatment-free interval & $\begin{array}{l}\text { HR, } 0.83 \text { ( } 95 \% \text { C } 1,0.46-1.52) \\
\text { PFS, NR vs NR } \\
N=40 \text { vs } 94\end{array}$ & $\begin{array}{l}\text { HR, } 0.48 \text { (95\% Cl, } 0.25-0.91) \\
\text { PFS, } 9.0 \text { mo vs NR } \\
N=32 \text { vs } 42\end{array}$ \\
\hline
\end{tabular}

Figure 1. Subset analysis of MONARCH 3 (placebo vs abemaciclib). Abbreviations: HR, hazard ratio; NR, not reached; PFS, progression-free survival.

Adapted from Goetz MP, O'Shaughnessy J, Sledge Jr GW, et al. The benefit of abemaciclib in prognostic subgroups: an exploratory analysis of combined data from the MONARCH 2 and 3 studies [abstract]. Presented at the 2017 San Antonio Breast Cancer Symposium; December 5-9, 2017; San Antonio, Texas. Abstract GS6-02.
$P=.0000000983)$. Choice of the endocrine agent did not influence outcomes.

Although their efficacy appears the same, the 3 agents do have some differences in schedule of administration and toxicity profile (Figure 2).

\section{Fulvestrant Finds a Place in the Guidelines}

Enhancing endocrine therapy may be possible, even without adding a CDK4/6 inhibitor, Dr. Gradishar said. Fairly recent data come from the first-line FAL$\mathrm{CON}$ trial of anastrozole versus fulvestrant at 500 mg monthly. ${ }^{18}$ PFS was significantly longer in the fulvestrant group ( $\mathrm{HR}, 0.79 ; P=.0486$ ), with a median PFS of 16.6 versus 13.8 months for anastrozole. In the absence of visceral disease, the difference was more striking, with an absolute benefit of at least 8 months, he added.

"The findings suggest that giving fulvestrant at $500 \mathrm{mg}$ a month with a loading dose as well, may actually enhance PFS over anastrozole alone," he commented. "So there are a number of different options that one could consider in the absence of a CDK4/6 or mTOR inhibitor [everolimus]."

\section{A Role for Extended Therapy?}

The NCCN Guidelines for Breast Cancer indicate that women who are postmenopausal at diagnosis can consider extending endocrine therapy for an additional 5 years. "For instance, one could consider extending tamoxifen to 10 years, or you can sequence tamoxifen with an AI. You could start with an AI, and then decide that you're going to continue that beyond 5 years," Dr. Gradishar explained.

The data to support longer duration of treatment rely largely on a pivotal paper published in 2017 by Pan et al. ${ }^{19}$ They performed a meta-analysis on 88 trials involving 62,923 women with ER-positive breast cancer who were disease-free after 5 years of endocrine therapy, and found that breast cancer recurrences continued to occur steadily throughout the study period, from 5 to 20 years. The risk of distant recurrence was strongly correlated with the original TN status.

"After 5 years, regardless of whether the patient was node-negative or -positive, even in the bestprognosis patients, there was an increased risk for recurrence out to 20 years," Dr. Gradishar noted. Risk for distant recurrence at 20 years was $22 \%$ for patients who were node-negative, $32 \%$ for patients 
NCCN Guidelines Updates: Breast Cancer

\begin{tabular}{|c|c|c|c|}
\hline Toxicity & Palbociclib & Ribociclib & Abemaciclib \\
\hline Dosing schedule & 3 wk on, 1 wk off & 3 wh on, 1 wk off & Continuous \\
\hline $2 G$ rade 3 neutropenia & $66 \%$ & $59.6 \%$ & $21.1 \%$ \\
\hline Febrile neutropenia & $1.6 \%$ & $1.5 \%$ & $<\%$ \\
\hline$\geq$ Grade 3 diarrhea (all grade) & $186(26 \%)$ & $1.2 \%(35 \%)$ & $9.5 \%(81 \%)$ \\
\hline Grade 2/3 OTC prolongation & - & 310.3 & - \\
\hline ZGrade 3 AST/ALT increase & . & $5.79 .3 \%$ & $3.87 \%$ \\
\hline $\begin{array}{l}\text { Dose reduction/discontinue due } \\
\text { to AEs }\end{array}$ & $36 \% / 9.7 \%$ & $51 \% / 7.4 \%$ & $43.4 \% / 19.6 \%$ \\
\hline Alopecia & 33\% & 33\% & $27 \%$ \\
\hline Increased creatinine & - & . & $98 \%(n) f(n)$ \\
\hline VTEPE & $0.9 \%$ vs $1.4 \%$ & NR & $4.9 \%$ vs $0.6 \%$ \\
\hline
\end{tabular}

Figure 2. Differences in dosing and toxicity profiles of first-line treatment with palbociclib, ribociclib, and abemaciclib.

Abbreviations: AE, adverse events; ALT, alanine transaminase; AST, aspartate aminotransferase; NR, not reported; PE, pulmonary embolism; VTE, venous thromboembolism.

with 1 to 3 positive nodes, and 52\% for patients with 4 to 9 positive nodes. "This begs the question of whether continued therapy beyond that 5 years might be prudent for some patients," he said.

In almost 10 large studies examining the benefit of extended-duration endocrine therapy, the reduction in DFS risk has varied widely, and overall survival has not been affected. Altogether these studies are "sort of a mixed bag," Dr. Gradishar acknowledged, "but perhaps an incremental improvement in outcome does occur with longer durations of therapy."

Several recent data sets (all first reported in 2016) either clarify the issue or confuse it a bit. The MA17R trial examined 5 versus 10 years of letrozole after 5 years of tamoxifen. "You can see the curves- with a magnifying glass," Dr. Gradishar commented. "From a statistical standpoint, there probably is an incremental improvement, but it really is in the prevention of contralateral breast cancer. And with the cost of the side effects that come with longer durations of an AI."

NSABP B-42 also examined longer duration of an $\mathrm{AI}$ and found very modest benefit, primarily seen in the breast cancer-free interval (HR, 0.71; $P=.003) .{ }^{21}$ The DATA trial found some improvement in DFS (HR, 0.79; $P=.007$ ), ${ }^{22}$ but the IDEAL trial did not (HR, 0.96; $P=.07 ; P=.59$ ), ${ }^{23}$ and neither showed a survival difference. The ABCSG-16 trial with 3,484 patients also showed no difference in DFS but did show much more toxicity with extended therapy. ${ }^{24}$

"What you do see, with time, is that with longer durations of therapy you are also buying more of the side effects-more osteoporotic fractures and an incremental increase in the risk of thrombotic events," he added.

"Would I say that these data suggest that no patient should receive longer durations of therapy? No, but it really emphasizes the need to individualize," Dr. Gradishar concluded. "We have to be cognizant, as well, of what comes with longer durations. You have to get a patient out to 5 years before you could even consider longer treatment, and she has to be absent the significant musculoskeletal complaints, and the concerns about osteoporosis, changes in lipid profile, and possibly hypertension. Decisions about duration must be individualized."

\section{References}

1. von Minckwitz G, Procter M, de Azambuja E, et al. Adjuvant pertuzumab and trastuzumab in early HER2-positive breast cancer. N Engl J Med 2017;377:122-131.

2. Chan A, Delaloge S, Holmes FA, et al. Neratinib after trastuzumab-based adjuvant therapy in patients with HER2-positive breast cancer (ExteNET): a multicentre, randomised, double-blind, placebo-controlled, phase 3 trial. Lancet Oncol 2016;17:367-377.

3. Tolaney SM, Barry WT, Dang CT, et al. Adjuvant paclitaxel and trastuzumab for node-negative, HER2-positive breast cancer. N Engl J Med 2015;372:134-141.

4. Tolaney SM, Barry WT, Guo WT, et al. Seven-year followup of adjuvant paclitaxel and trastuzumab (APT trial) for node-negative, HER2-positive breast cancer [abstract]. J Clin Oncol 2017;35(Suppl 15):Abstract 51.

5. Byrski T, Huzarski T, Dent R, et al. Pathologic complete response to neoadjuvant cisplatin in BRCA1-positive breast cancer patients. Breast Cancer Res Treat 2014;147:401-405.

6. Tutt A, Ellis P, Kilburn L, et al. The TNT trial: a randomized phase III trial of carboplatin (C) compared with docetaxel (D) for patients with metastatic or recurrent locally advanced triple negative or BRCA $1 / 2$ breast cancer (CRUK/07/012) [abstract]. Cancer Res 2015;75(Suppl):Abstract S3-01.

7. Robson M, Im SA, Senkus E, et al. Olaparib in metastatic breast cancer in patients with a germline BRCA mutation. N Engl J Med 2017;377:523533.

8. Adams S, Loi S, Toppmeyer D, et al. Phase 2 study of pembrolizumab as first-line therapy for PD-L1-positive metastatic triple-negative breast cancer (mTNBC): preliminary data from KEYNOTE-086 cohort B [abstract]. J Clin Oncol 2017;35(Suppl):Abstract 1008.

9. Dirix LY, Takacs I, Jerusalem G, et al. Avelumab, an anti-PD-L1 antibody, in patients with locally advanced or metastatic breast cancer: a phase $1 \mathrm{~b}$ JAVELIN Solid Tumor study. Breast Cancer Res Treat 2018;167:671-686.

10. Nanda R, Liu MC, Yau C, et al. Pembrolizumab plus standard neoadjuvant therapy for high-risk breast cancer (BC): results from I-SPY 2 [abstract]. J Clin Oncol 2017;35(Suppl):Abstract 506.

11. Modi S, Pusztai L, Forero A, et al. Phase 1 study of the antibody-drug conjugate ladiratuzumab vedotin (SGN-LIV1A) in patients with heavily pretreated triple-negative metastatic breast cancer [abstract]. Presented at the 2016 San Antonio Breast Cancer Symposium; December 5-9, 2017; San Antonio, Texas. Abstract PD3-14.

12. Bendell J, Saleh M, Rose AA, et al. Phase I/II study of the antibody-drug conjugate glembatumumab vedotin in patients with locally advanced or metastatic breast cancer. J Clin Oncol 2014;32:3619-3625.

13. Yardley DA, Weaver R, Melisko ME, et al. EMERGE: a randomized phase II study of the antibody-drug conjugate glembatumumab vedotin in advanced glycoprotein NMB-expressing breast cancer. J Clin Oncol 2015;10;33:1609-1619.

14. Bardia A, Mayer IA, Diamond JR, et al. Efficacy and safety of anti-trop-2 antibody drug conjugate sacituzumab govitecan (IMMU-132) in heavily 
Giordano et al

pretreated patients with metastatic triple-negative breast cancer. J Clin Oncol 2017;35:2141-2148

15. Traina TA, Miller K, Yardley DA, et al. Enzalutamide for the treatment of androgen receptor-expressing triple-negative breast cancer. J Clin Oncol 2018;36:884-890.

16. Goetz MP, O'Shaughnessy J, Sledge Jr GW, et al. The benefit of abemaciclib in prognostic subgroups: an exploratory analysis of combined data from the MONARCH 2 and 3 studies [abstract]. Presented at the 2017 San Antonio Breast Cancer Symposium; December 5-9, 2017; San Antonio, Texas. Abstract GS6-02.

17. Tripathy D, Sohn J, Im SA, et al. First-line ribociclib or placebo combined with goserelin and tamoxifen or a non-steroidal aromatase inhibitor in premenopausal women with hormone receptor-positive, HER2negative advanced breast cancer: results from the randomized phase III MONALEESA-7 trial [abstract]. Presented at the 2017 San Antonio Brest Cancer Symposium; December 5-9, 2017; San Antonio, Texas. Abstract GS2-05.

18. Robertson JF, Bondarenko IG, Trishkina E, et al. Fulvestrant $500 \mathrm{mg}$ versus anastrozole $1 \mathrm{mg}$ for hormone receptor-positive advanced breast cancer (FALCON): an international, randomised, double-blind, phase 3 trial. Lancet Oncol 2016;388:2997-3005.
19. Pan H, Gray R, Braybrooke J, et al. 20-year risks of breast-cancer recurrence after stopping endocrine therapy at 5 years. N Engl J Med 2017;377:18361846.

20. Goss PE, Ingle JN, Pritchard $\mathrm{KI}$, et al. Extending aromatase-inhibitor adjuvant therapy to 10 years. N Engl J Med 2016;375:209-219.

21. Mamounas EP, Bandos H, Lembersky BC, et al. A randomized, doubleblinded, placebo-controlled clinical trial of extended adjuvant endocrine therapy with letrozole in postmenopausal women with hormone-receptorpositive breast cancer who have completed previous adjuvant treatmen with an aromatase inhibitor: results from NRG Oncology/NSABP B-42 [abstract]. Cancer Res 2017;77(Suppl):Abstract S1-05.

22. Tjan-Heijnen VCG, van Hellemond IEG, Peer PGM, et al. Extended adjuvant aromatase inhibition after sequential endocrine therapy (DATA) a randomized, phase 3 trial. Lancet Oncol 2017;18:1502-1511.

23. Blok EJ, Kroep JR, Meershoek-Klein Kranenbarg E, et al. Optimal duration of extended adjuvant endocrine therapy for early breast cancer; results of the IDEAL trial (BOOG 2006-05). J Natl Cancer Inst 2018;110:40-48.

24. Gnant M, Pfeiler G, Dubsky PC, et al. The impact of adjuvant denosumab on disease-free survival: results from 3,425 postmenopausal patients of the ABCSG-18 trial [abstract]. Cancer Res 2016;76(Suppl):Abstract S2-02. 